\title{
Exploring the constraints regarding maternal health in reproductive age among the rural women in Bangladesh
}

\author{
AC Das
}

\begin{abstract}
The study evaluated the constraints of maternal health in reproductive age in the rural Bangladesh. The study used qualitative approach to gather information where individual in-depth interviews adopted for data collection among women aged 15-49 years old. The overall study revealed that rural women faced many maternal health related complications and problems in their reproductive age such as hemorrhage, sepsis, hypertensive diseases of pregnancy, obstructed labour and complications of abortion, etc. Maternal health situation still in rural areas was not developed in comparison to the situation of urban areas of Bangladesh and there was not available women's health care accesses for their emergency; low education level, low per capita income, many family members, early marriage and pregnancy, number of pregnancies, poor nutrition and lack of family support status resulted in low status of maternal and child health in the rural areas of Bangladesh. Adequate measures should be taken for providing proper health care services in rural areas of Bangladesh for better maternal health status.
\end{abstract}

Key words: Women's health, reproductive age, pregnancy, abortion, Bangladesh.

\section{Introduction}

Maternal health includes pre-conception care, antenatal care, safe delivery care, emergency obstetric care, postnatal care, care of the new born and family planning. ${ }^{1} \mathrm{~A}$ maternal death is defined as the death of a woman while pregnant or within forty two days of termination of pregnancy, irrespective of the duration and the site of the pregnancy. ${ }^{2}$ Maternal mortality and morbidity remain high in Nepal, India, Bangladesh and Pakistan, and policy in the region has focused increasingly on skilled attendance at birth to reach Millennium Development Goal 5 (MDG5). ${ }^{3-5}$ In all four countries, use of maternal health care services is limited despite increased inputs from governments and international donors. ${ }^{6}$ Though use of antenatal care, skilled birth attendance and emergency obstetric care are mediated by a range of factors, there is increasing evidence that financial and other barriers are important in determining service uptake. ${ }^{3,7}$ Complications during pregnancy and childbirth such as haemorrhage, sepsis, spontaneous abortion, pre-eclampsia and eclampsia, and obstructed labour are the leading causes of death and disability among women of reproductive age in developing countries. ${ }^{8}$ Many deliveries still occur at home and without the assistance of trained attendants in low and middle-income countries. ${ }^{9-12}$ Women in Bangladesh have risk of dying from the causes of pregnancy and childbirth and many of 
them died due to maternity-related causes. ${ }^{13}$ However, like many other developing countries among the major underlying factors leading to poor maternal situation in Bangladesh included very low percentages of women actually seek professional medical assistance for pregnancy related care, deliveries and complications. ${ }^{14}$ Although Bangladesh made commendable improvements in uptake of maternal care services over the period between 1995 and 2010, still there is the very low utilization of antenatal care, postnatal care and skilled birth attendance among poorer and less-educated, as well as women living in rural areas, remains a major impediment to further improvement in reproductive, maternal and child health outcomes. ${ }^{15}$ The World Health Organization defines skilled care as 'quality care' to a woman during pregnancy, childbirth and postpartum period by a skilled personnel supported by an enabling environment (necessary equipment, supplies and medicines and infrastructure) and a functional referral system. ${ }^{16}$ At the centre of skilled care is a skilled attendant who is a health professional (midwife, nurse, doctor) educated and trained to proficiency in the skills needed to manage normal pregnancy, childbirth and the immediate post-partum and in the identification and management or referral of complications. ${ }^{16}$ The fee free policy aligns with other international strategies to reduce maternal mortality rates including the MDG5 that called for a reduction in maternal mortality by three quarters by 2015 . $^{16,17}$

Reducing maternal and child mortality requires focused attention on better access, utilization and coverage of good quality health services and interventions aimed at improving maternal and newborn health among target populations, in particular, pregnant women where antenatal care is a key strategy for reducing maternal and neonatal morbidity and mortality rate because adequate utilization of antenatal health care services is associated with improved maternal and neonatal health outcomes. ${ }^{18,19}$ To improve priority behaviors, messaging and media strategies addressed the most salient behavioral determinants through face-to-face communication, social mobilization and mass media exposure, which play a critical role in rapidly reaching mothers, household members, community influential and health workers on a large scale; they were effective in improving infant and young child feeding practices. ${ }^{20}$ In Bangladesh, maternal health situation is still lower among the women in rural areas. Studies conducted so far on maternal health situation in rural areas of Bangladesh have lacking of observing the maternal health situation in reproductive age along with complications and problems. The aim of this study is to fill this gap. This study examines the prevalence of maternal health in their reproductive age and its constraints among rural women in Bangladesh and offers recommendations for mitigating the associated factors.

\section{Materials and Method \\ Study design}

The design was a descriptive qualitative study. Face-to-face In-Depth Interviews (IDIs) were conducted among the rural women who had at least one delivery experience aged 15-49 years old. The interviews were conducted in the local language, Bangla, using a semi-structured interview guide. Throughout the interviews, follow-up questions using probes were asked in order to acquire a deeper understanding when an explanation was unclear. Each of the interviews lasted on average 45 minutes.

\section{Setting}

The study was done among the women of seven sub-districts (Bhola Sadar, Burhanuddin, Charfasson, Daulatkhan, Lalmohan, Manpura, Tazumuddin) of Bhola district in Bangladesh where health conditions and socioeconomic status were very low. Twenty three IDIs were conducted from October to December, 2015 and stopped data collection because of saturation of information.

\section{Data collection}

A qualitative approach was adopted for data collection. IDIs were used to generate information. At the beginning after greetings and formalities for consent form, questions were asked to understand more deeply women's 
perceptions of maternal health care with the association of maternal and child health. Interviews were performed in local languages by two assistants. All interviews were audio-recorded except for two participants who refused to have their responses audio-taped. For these two participants, hand written notes were used to record responses. Field records were taken for all participants' responses and the hand written notes were expanded into transcripts.

\section{Data analysis}

All of the collected interviews were transcribed and translated in English, prepared and analyzed by using MAXQDA 10.0, which is a qualitative data management software. The completed interviews were transcribed from the digital recording and translated into English by two experts. The transcripts were de-identified by the research team before any coding activity took place. Utilizing an inductive approach to coding, three research staffs coded the transcripts, discussing and editing themes as they emerged, and developed a coding template. The data were analyzed using the thematic content analysis method.

\section{Ethical consideration}

Oral inform consent was obtained in the study on the knowledge of maternal health among general population. Participants who agreed to participate in this study signed an informed consent form or gave a thumb print if they were illiterate. The study was approved by Department of Population Sciences, University of Dhaka, Bangladesh. Participation in the study was voluntary and participants were assured that anonymity would be observed at all times. Confidentiality of participants was maintained by using numbers on both the recorded interviews and transcripts.

\section{Results}

Table 1 shows the socio-demographic profile of study participants. Fig. 1 shows the type of family (extended and nuclear) of the women. Fig. 2 presents the occupations of husbands of the women.

The study revealed that there are a lot of problems and complications related to mater- nal health during pre-conception, pregnancy, delivery, postnatal and childbearing. It was also found that maternal healthcare situation of women in rural Bangladesh was comparatively developed than that of the last few decades had but still many complications identified in the rural areas of Bangladesh and these resulted the rate of higher maternal mortality and morbidity in their maternal period. In rural Bangladesh women are facing difficulties in the different time those are isolated by pre-conception, pregnancy, delivery, postnatal and childbearing period to observe the maternal health situations. The key findings of this study are presented in the Table 2 .

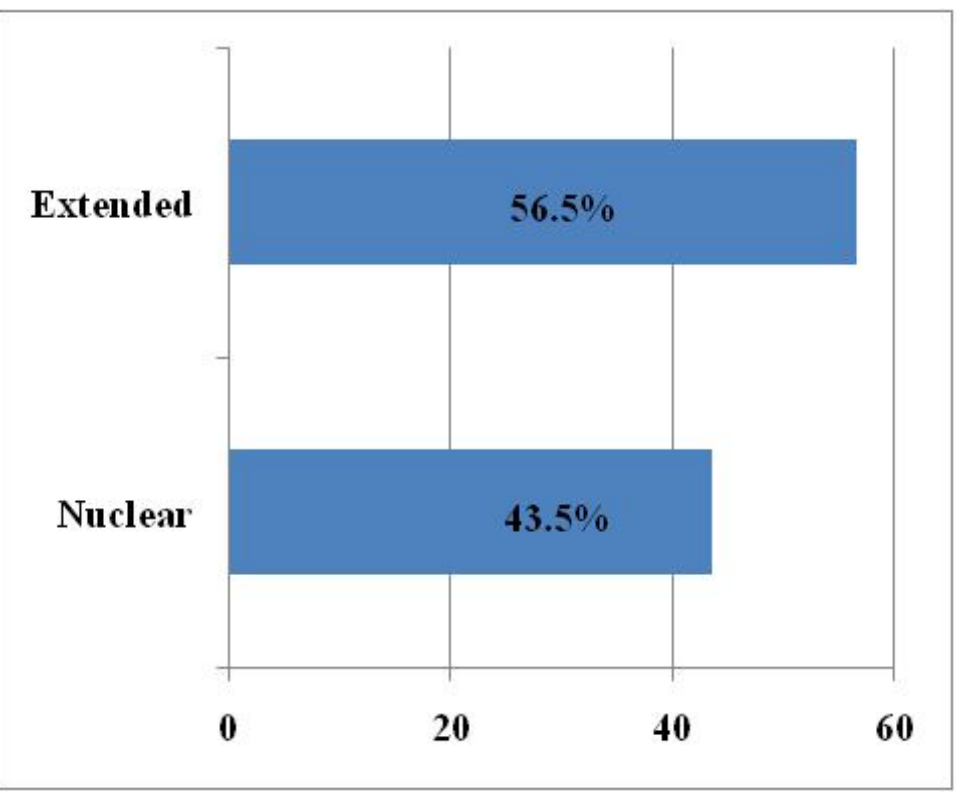

Fig. 1. Type of family of the women.

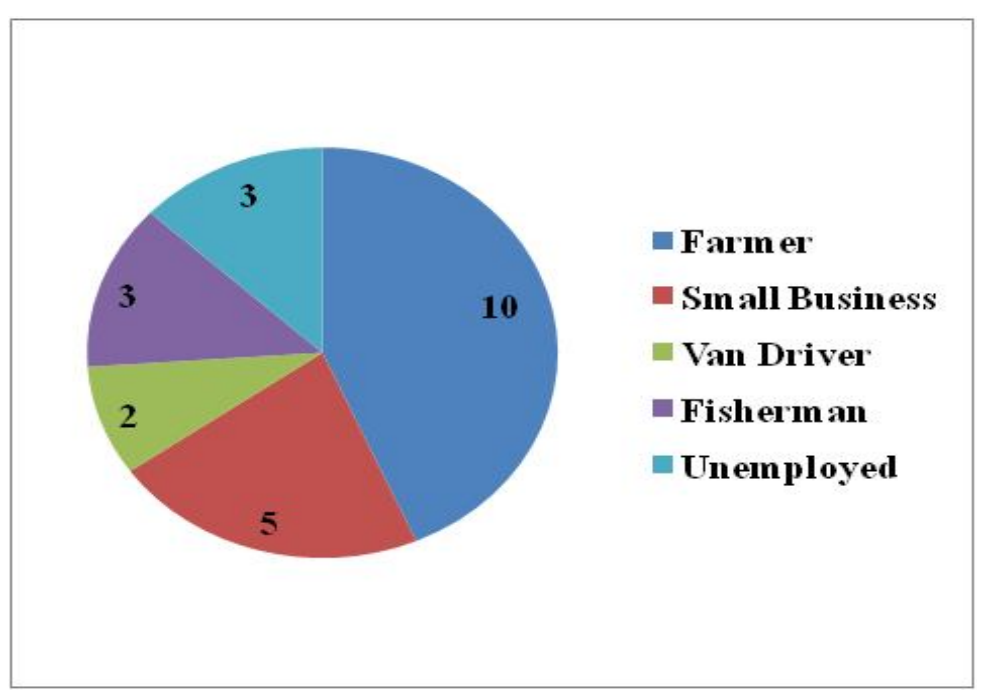

Fig. 2. Husband's occupation. 
Table 1. Socio-demographic profile of study participants

\begin{tabular}{lll}
\hline Characteristics & Mean \pm SD & Range \\
\hline Women's information & & \\
Current age (years) & $24.6 \pm 5.0$ & $16-35$ \\
Age at marriage (years) & $14.0 \pm 1.7$ & $11-17$ \\
Education (years) & $5.5 \pm 3.6$ & $0-10$ \\
Number of pregnancy & $2.3 \pm 0.8$ & $1-4$ \\
Number of alive children & $2.0 \pm 0.7$ & $1-3$ \\
Husband's Information & & \\
Current age (years) & $30.6 \pm 4.8$ & $24-40$ \\
Age at marriage (years) & $21.1 \pm 2.5$ & $17-26$ \\
Education (years) & $5.6 \pm 4.2$ & $0-12$ \\
\hline
\end{tabular}

Table 2. Summary of key findings

\begin{tabular}{ll}
\hline Concerned periods & Summary of key findings reported by the women \\
\hline Pre-conception period & - did not do necessary medical tests \\
& - did not take proper food and treatment because of lower family \\
financial condition & - faced reproductive health related complications \\
\hline Pregnancy period & - lack of awareness aboutpregnancy and maternal health care \\
- most common complications were maternal bleeding, complications & of abortion, high blood pressure of pregnancy, maternal sepsis, \\
& tiredness, constipation, pelvic girdle pain, back pain and obstructed \\
& labour, etc \\
- swelling of hands and face, irregular and painful bowel and urination & - lack of supports provided during pregnancy \\
\hline - did not receive necessary health facilities and delivery supports \\
- lack of proper treatment of the complications \\
- did not go to qualified doctors or health professionals \\
- lack of knowledge and awareness about healthcare \\
- much reproductive organ related problems but did not have enough \\
treatment
\end{tabular}

Pre-conception period

Majority of the participants reported that women faced many maternal health related complications and problems in their pre-conception period; it was noted that pre-conception care improves women's chances of falling pregnant more easily, having a healthy pregnancy and healthy baby and aiding heip quick recovery after the delivery. The study found that the rural women did not do the blood tests that may include a full blood count and ferritin levels and a test to see whether they were immune to rubella or not. Further a test to check urine for infection, protein and glucose, dental check up, and a blood pressure check were not done among them to ensure the normal range. And unfortunately, as these problems 
were not properly identified in the time of pre-conception among the rural women in Bangladesh, they faced not only these problems or complications but also suffered much. One (IDI-5) of them reported that "Chicken pox was in my whole body after four-five months of pregnancy and they were so painful. The rashes appeared again after delivery so that I did not breastfeed my child properly because of chicken pox. On the other hand, there was problem in my uterus." Another respondent (IDI-23) reported that "I have one child but another child died during delivery when I was only 12 years and my husband was 18 years old. After three years of my marriage, first time I became pregnant but I did not take proper food because of low family financial condition so that the situation always kept all of us much in mental stress."

Folic acid, universally recommended supplement during pregnancy, is needed for the healthy growth and development of the baby in the first weeks of life. By taking folic acid supplement, research has found that the risk of birth defects such as spina bifida is reduced. In the rural areas of Bangladesh, there is still a lot of lackings among the women in taking proper healthy food and treatment so that they are facing different pre-conception related complications and problems. One (IDI-9) of the women reported that "I am 30 years and my husband is 32 years older. We have been married for past 5 years. We have been trying for a baby past 12 months but unsuccessful and now I have got problems with my menstrual cycle. It was never regular but now it is worst. I don't get my periods for 3 months. I feel obsessed and irritated. Consulted a Gynae specialist before 2-3 months and am under medication, but still the situation is not good."

\section{Pregnancy period}

Complications of pregnancy are health problems that are caused by pregnancy. There is no clear distinction between complications of pregnancy and symptoms and discomforts of pregnancy. However, the latter do not significantly interfere with activities of daily living or pose any significant threat to the health of the mother or baby. The women reported in this study that the most common causes were maternal bleeding, complications of abortion, high blood pressure of pregnancy, maternal sepsis, tiredness, constipation, pelvic girdle pain, back pain and obstructed labour, etc. A young woman (IDI-17) reported that "Uterus got blocked so that there was no birth attendant who encouraged assisting in the time of doing delivery; then took a skilled birth attendant who took training on it. However, my husband was sent to take homeopathy because the baby could not be delivered but when he was in the middle of the path of coming home passing 1-2 hours, the delivery took place." On the other hand, it was narrated by a participant (IDI-2) that "In my pregnancy time, I couldn't walk and did not up and down, had bleeding from nose and mouth."

Most of the participants of the study gave reasons for failure to wait for delivery at health facility or a suitable place because some of the reasons were feared of people who could bewitch a pregnant women not to deliver on time even after the onset of labour, responsibilities such as looking after an elderly mother who was at home alone that made it difficult to leave and lack of a guardian. Relatives were reluctant to be guardians at the health facility as they were busy in their personal works. It has been narrated as follows: "IDI-20: I faced many complications but didn't go to a doctor for treatment because of their poverty; a birth attendant suggested not to be frustrated and to depend on God who will save. I was unable to work in that time she helped me but there was not any of our relatives who helped us" and "IDI-19: During my first pregnancy, I faced pain in spine and lower abdomen, and problem in urination. And there was pain like delivery, which was six months before delivery and continued up to delivery." Another woman (IDI-3) explained "When I was pregnant, I suffered from tremor, vertigo, and headache and laid down, blurred vision, generalized weakness, 
etc but unfortunately I did not receive any kinds of supports from anyone or did not get proper treatment."

However, a few participants reported that they faced many problems in the time of their pregnancy. Pneumonia and excessive vomiting were very common names of the complications in their area among the women when they become pregnant. Some of them reported: "IDI-4: I was suffering from pneumonia. Again and again, coughing was with blood. Vomiting was another reason, which affected my health and I stopped eating except water." "IDI-16: When I became pregnant, I faced many problems. I could not eat any food because of having much vomiting and excessive headache." "IDI-1: After becoming pregnant, I faced different kinds of problems such as swelling of hands and face, irregular and painful bowel and urination. I couldn't eat properly and faced much headache." "IDI-12: I found tastelessness in rice and fish when I became pregnant so that I was unable to eat enough and I couldn't move from here and there because of my weakness."

In addition, pregnancy may result in pregnancy related complications such as deep vein thrombosis or worsening of an intercurrent disease in pregnancy. But family support and vulnerability are also the reasons that make the much suffering of the rural women's maternity and childbearing. Few of the women answered: "IDI-16: My husband hurt me in pregnancy time so that there were many problems I had to face. I couldn't move here and there because of feeling pain in my uterus. Sometimes, I felt that delivery will be now but wouldn't." "IDI-14: In my last pregnancy, I had medicine from a pharmacy of village market because of facing severe bleeding and after six months I went to a doctor of district hospital where I received the treatment and we had to spend much money although the financial condition of my husband was not sufficient."

\section{Delivery period}

The health facility centers or hospitals are not available in all of the rural areas of Bangladesh. A few participants reported that labour started at night so that some of them started off for the health facility but delivered in transit, while others stayed and delivered in their homes. One (IDI-1) of them narrated that "I faced very terrible situation when I gave birth to my first child. Almost I have been saved from the death. Three days after suffering, I delivered the child getting the help of a doctor, who was taken from the sub-district, Daulatkhan, taking loan from one of our neighbours." Another woman (IDI-8) explained that "I suffered a lot of problems so that admitted in hospital because of severe bleeding and also breastfeeding problem; felt mass in my breasts. I stayed one month at hospital but the problem was not been solved; then a local doctor from sub-district treated and I became cured."

A few of the respondents reported that "IDI-11: I faced pain in uterus and leucorrhoea aggravated with cough." "IDI-3: During delivery time, I suffered a lot of headache and sometimes I laid down; I delivered a child after getting pain up to five days."

Other women reported the extreme worst experiences in their delivery time that "IDI-3: I felt pain in the uterus and thought I did not face this kind of problems when I gave birth to last three children, I faced these problems during my last child birth. My uterus prolapsed out with bleeding and leucorrhoea aggravated with coughing; I became senseless. Surgery was done to out the placenta but still suffering much because of it." "IDI-4: In the time of my first child, leucorrhoea problem was before delivery and I fell down three months back; then a dead child was born. And also my other child had died because of pneumonia in the time of my third pregnancy." "IDI-22: In the time of my second child to be delivered, I was so weak and always thought that I am going to die when I was pregnant for three months; then after five to six months the baby was delivered when I was suffering from fever."

The women of the area more faithfully went to the traditional healers for the treatment of complications faced in their maternal time because of having much comfortable treatment with the lowest cost. A participant 
(IDI-15) explained her experiences that "My first child was born dead because the position of my child reversed during the delivery time. Another child had also died after birth. In the time of my third child to be delivered, I almost was saved from the death because of facing many complications and my baby's health condition also was very bad but going to a traditional healer saved my life."

\section{Postnatal period}

Almost all of the participants reported that they faced many complications in their postnatal period. One (IDI-5) of them said that "After giving birth to my fourth child, I was vomiting and vomiting so that I became almost senseless and then faced convulsions on my hands and feet." A Muslim woman (IDI-6) reported that "In all of my childbearing time, I faced many problems and difficulties; last one was induced abortion when I was pregnant for three months. There had a problem with uterus, which was getting out when try to seat. Bleeding happened every month two or three times so that I couldn't do my prayer properly." Another woman (IDI-14) explained that "Headache and pain in my womb but only used herbs for relieve from the pain. It was not possible to examine during any of the pregnancies because my husband and mother-in-law didn't allow it." On the other hand, a woman (IDI-19) informed that "Seven days after suffering from much pain and different complications, I delivered my baby when there started delivery pain an injection was given by a local doctor for starting proper delivery pain."

A few women explained their experiences of facing different complications and problems in their postnatal time as follows: "IDI-4: My husband did not have much money; so took the help of a traditional healer and had medicine from a pharmacy of local market and I was treated." "IDI-10: Traditional healer is one of the most trusted persons to whom I interestingly like to go for treatment because of better treatment and also it must be mentioned that my child has been saved from pneumonia by taking the treatment from a traditional healer near our house." "IDI-15: Doctors and hospitals are very far so that we did not get proper treatment and helps on time. During my second child to be delivered, I faced a lot of problems so that doctors were called but my child had died before the doctor came." "IDI-17: Birth attendant suggested to take homeopathy pain killer after delivery and also vitamins from our local market by the advice of birth attendant."
Childbearing period

Most of the participants narrated that after the delivery of a baby they were neglected by other family members and also husband to take care so that the health of mother and child became much vulnerable and as a result, the situation becomes an extreme worsen. When the rural women were facing numerous complications and problems regarding their health, they explained the situation to their husband and mother-in-law but they did not take it seriously so that their sufferings increased day by day. A participant (IDI-5) pointed that "In the time of my fourth childbearing, I suffered from vomiting a lot so that I could not keep anything in my stomach and many times I became senseless having convulsions; however, I did not get proper treatment and support." A middle aged woman (IDI-16) reported that "My husband and other family members are not enough educated so that they do not understand that my new born baby and I needed help from the proper health care provider and also need health care facilities and healthy food but unfortunately they did not allow me; I wanted and always said for going to a male doctor but it was considered as going against our religion."

Few women explained the situation of her family income and sufferings where many times they were silent after all they needed full health care support. A respondent (IDI16) reported that "If I told to my husband to buy medicine he would buy but I didn't want to tell because I knew what condition we were facing, even many times we all did not have enough food." The rural women are not able to go to a doctor. After all, having government health care centres or hospitals beside them, they believe and feel comfort going to a traditional healer. One (IDI-2) of the participants explained the situation she faced that there are a lot of problems in her maternal time as "I faced severe pain and I did not see any doctor because of facing financial problems so that I treated the problems by a traditional healer and saved my life." Another woman (IDI-23) narrated that "We are poor so that we are unable to go to a doctor when facing a lot of problems in our maternal time but it is easy and comfortable to get treatment from a traditional healer than a doctor. Interestingly, in my opinion, they are more expert comparing to the available doctors. Most of the women of our area firstly choose to see a traditional healer for saving time and money." 
Discussion

Bangladesh is one of the few developing countries that achieved the MDG5 in time, despite skilled birth attendance remaining low. ${ }^{21}$ However, emergency transport and communication is an important part of all emergency health services, specifically for reduction of maternal mortality and it has also found by this study that transport and communication problems created a lot of dangerous situations in the time of maternity in rural Bangladesh. ${ }^{22}$ Improving social accountability in maternal health services requires intervention by creating spaces where health providers and women are enabled to have constructive dialogue, and health providers can be more receptive to discuss health concerns or demands made by women. ${ }^{23}$ During delivery time, there are a lot of complications and problems faced by the rural women such as hemorrhage, sepsis, hypertensive diseases of pregnancy, obstructed labour and complications of abortion; still these are the reasons of higher maternal mortality ratio in Bangladesh where the utilization of maternity care provided by trained professionals during and after delivery is alarmingly low, primarily due to lack of knowledge and money. ${ }^{24}$

On the other hand, the status of rural Bangladeshi adolescent girls' awareness about health such as reproductive health, reproduction, sexually transmitted diseases, and HIV/AIDS is very poor and they even have incorrect knowledge or misconceptions about the fertile period. It has found by another study that improved access to mass media and education could improve rural Bangladeshi adolescent girls' awareness about reproductive health. ${ }^{25}$ In health sector, antenatal care is an important determinant of reducing high maternal mortality rate and one of the basic components of maternal care on which the life of mothers and babies depends mostly. A number of studies have identified lack of antenatal care as a risk factor for maternal mortality and there is the low proportion of women who deliver with a skilled birth attendant. ${ }^{26,27}$ The skilled midwives were well accepted in the community but discouraged by center authority for home birth, apprehending that it will reduce their client flow and earnings but noncooperation by other health care providers was common. ${ }^{28}$

The study pointed that suffering in the time of pregnancy period is much higher among the rural women who don't have enough education and family income than those who had higher per capita income and education. Education, number of living children, and current contraceptive use were important predictors of exposure to any mass media family planning messages but there was a negative relationship between breastfeeding and the current use of contraception indicating a low need for contraception among women who breastfed and utilization of maternal health services remains low in the low-resource countries like Bangladesh and the women reported that still there is low utilization of antenatal care. ${ }^{29,30}$ For these reasons, the priority focus should be given on implementing and evaluating multi-sectoral interventions to improve access to and quality of care for women who are poorer, less-educated and live in rural areas in the post-MDG health and development agenda for achieving universal health coverage, as there is considerable potential for improvement among these groups. ${ }^{15}$ Another study found that the proportion of women who received antenatal care services and information on the signs of pregnancy complications as well as the median number of antenatal care visits showed an increase in maternal health status. ${ }^{30}$

\section{Limitations}

The study might suffer a bias because the participants were interviewed on the premises of the husband's house, where they had to follow their roles so that they possibly were not able to give most important information related to this study. Some of these women had multi-pregnancy or delivery experiences and they might have included experiences from previous pregnancies resulting in some recall bias when the questions asked about the last pregnancy experiences. 


\begin{abstract}
Conclusions
The overall finding of this study indicates that maternal health situation in reproductive age is still low in rural Bangladesh and every year a large number of women suffered and died because of facing complications and problems such as hemorrhage, sepsis, hypertensive diseases of pregnancy, obstructed labour and complications of abortion, etc because of the very low utilization of antenatal care, postnatal care and skilled birth attendant among poorer and less-educated women. However, a few reasons such as low education level, low per capita income, many family members, early marriage and pregnancy, number of pregnancies, poor nutrition and lack of family support resulted in much lower maternal and child health status among rural women in Bangladesh. From the findings of the study, it is recommended that the government and non-government organizations should work mutually for providing proper health care services in rural areas of Bangladesh for better maternal health status.
\end{abstract}

\section{Acknowledgment}

The author of this study wishes to thank all of the study participants who worked selflessly. And the author is also very grateful to the people who directly and indirectly helped him conducting this study.

\section{Declaration of conflicting interests}

The author declared that there is no conflict of interests.

\section{References}

1. Michael GA, Kosia AM, Usman A, Mufunda J, Nyarang'o P. Knowledge, attitude and practice towards skilled care attendance among women of reproductive age group in Eritrea. J Eritrean Medi Assoc 2006;1(1):47-9.

2. Lancaster HO. Expectations of life: a study in the demography, statistics, and history of world mortality. Springer Science \& Business Media; 2012.

3. Schmidt JO, Ensor T, Hossain A, Khan S. Vouchers as demand side financing instruments for health care: a review of the Bangladesh maternal voucher scheme. Health Policy 2010;96:98-107.

4. Agha S. Impact of a maternal health voucher scheme on institutional delivery among low income women in Pakistan. Reprod Health 2011;8:4755-8.

5. Ensor T, Clapham S, Prasai DP. What drives health policy formulation: Insights from the Nepal maternity incentive scheme? Health Policy 2009;90:247-53.
6. McNamee P, Ternent L, Hussein J. Barriers in accessing maternal healthcare: evidence from low-and middle-income countries. Expert Rev Pharmacoecon Outcomes Res 2009;9(1):41-8.

7. Ensor T, Cooper S. Overcoming barriers to health service access: influencing the demand side. Health Policy Plan 2004;19:69-79.

8. World Health Organization. The World health report 2005: make every mother and child count: overview. 2005.

9. Mrisho M, Schellenberg JA, Mushi AK, et al. Factors affecting home delivery in rural Tanzania. Trop Medi Int Health 2007;12(7):862-72.

10. Bell J, Curtis SL, Alayón S. Trends in delivery care in six countries. 2003. http://www.popline.org/node/255979 (Accessed in March 25, 2016).

11. Kyomuhendo GB. Low use of rural maternity services in Uganda: impact of women's status, traditional beliefs and limited resources. Reprod Health Matters 2003;11:16-26.

12. Duong DV, Binns CW, Lee AH. Utilization of delivery services at the primary health care level in rural Vietnam. Soc Sci Med 2004;59:2585-95.

13. Barkat $A$, Rahman $M$, Bose $M L$, Com $M$, Akhter S. Modelling the first two delays of the "three-delays model" for emergency obstetric care in Bangladesh: a choice model approach. J Health Popul Dev Ctries 1997;1(1):57-67.

14. Rahman M, Tarafder T, Mostofa G. Modes of delivery assistance in Bangladesh. Tanzania J Health Res 2008;10(4):246-52.

15. Van de Poel E, Flores G, Ir P, O'Donnell O, Van Doorslaer E. Can vouchers deliver? An evaluation of subsidies for maternal health care in Cambodia. Bull World Health Organ 2014;92:331-9.

16. World Health Organization, ICM \& FIGO. Making pregnancy safer: the critical role of the skilled attendant. 2004.

17. Ministry of Health. National roadmap for accelerating the attainment of MDGs related to maternal and newborn health in Kenya. Nairobi: Reproductive Health, 2010.

18. Younes L, Houweling TA, Azad K, Costello A, Fottrell E. Estimating coverage of a women's group intervention among a population of pregnant women in rural Bangladesh. BMC Pregnancy Childbirth 2012;12:1-7.

19. Onasoga OA, Afolayan JA, Oladimeij BD. Factors influencing utilization of antenatal care services among pregnant 
women in Ife Central Lga, Osun State Nigeria. Adv Appl Sci Res 2012;3(3):1309-15.

20. Sanghvi T, Jimerson A, Hajeebhoy N, Zewale M, Nguyen GH. Tailoring communication strategies to improve infant and young child feeding practices in different country settings. Food Nutr Bull 2013;34:S169-80.

21. Prata N, Bell S, Quaiyum MA. Modeling maternal mortality in Bangladesh: the role of misoprostol in postpartum hemorrhage prevention. BMC Pregnancy Childbirth 2014;14:1-10.

22. Vora KS, Mavalankar DV, Ramani K, et al. Maternal health situation in India: a case study. J Health Popul Nutr 2009:184-201.

23. Mafuta EM, Dieleman MA, Hogema LM, et al. Social accountability for maternal health services in Muanda and Bolenge Health Zones, Democratic Republic of Congo: a situation analysis. BMC Health Serv Res 2015;15(1):1-17.

24. Rob U, Rahman M, Bellows B. Using vouchers to increase access to maternal healthcare in Bangladesh. Int Q Community Health Educ 2009-2010;30(4):293-309.

25. Uddin MJ, Choudhury AM. Reproductive health awareness among adolescent girls in rural Bangladesh. Asia Pac J Public Health 2008;20:117-28.

26. Nisar N, White F. Factors affecting utilization of antenatal care among reproductive age group women (15-49 years) in an urban squatter settlement of Karachi. JPMA J Pakistan Medi Assoc 2003;53:47-53.

27. Hussein J, Bell J, Nazzar A, Abbey M, Adjei S, Graham W. The skilled attendance index: proposal for a new measure of skilled attendance at delivery. Reprod Health Matters 2004;12:160-70.

28. Tasnim S, Rahman A, Shahabuddin A. Access to skilled care at home during pregnancy and childbirth: Dhaka Bangladesh. Int $Q$ Community Health Educ 2009-2010;30(1):81-7.

29. Kabir M, Islam MA. The impact of mass media family planning programmes on current use of contraception in urban Bangladesh. J Bio Sci 2000;32:411-9.

30. Hossain AH. Utilization of antenatal care services in Bangladesh: an analysis of levels, patterns, and trends from 1993 to 2007. Asia Pac J Public Health 2010;22(4):395-406.

\section{Suggestion for citation of the above:}

Das AC. Exploring the constraints regarding maternal health in reproductive age among the rural women in Bangladesh. Mediscope 2016;3(2):1-10. 